\title{
Realidade prática vivenciada pelos enfermeiros na classificação de risco em serviços
}

\section{de urgência e emergência}

\section{Practical reality experienced by nurses in risk classification in emergency and emergency services Realidad práctica experimentada por enfermeras en clasificación de riesgo en servicios de} emergencia y emergencia

Pedro Bezerra Xavier ORCID: https://orcid.org/0000-0002-4212-1551 Universidade Federal do Rio Grande do Norte, Brasil E-mail: pedrobx37@gmail.com

Ísis de Siqueira Silva

ORCID: https://orcid.org/0000-0002-2403-2504 Universidade Federal do Rio Grande do Norte, Brasil E-mail: isis1998.siqueira.silva@gmail.com

Jank Landy Simôa Almeida

ORCID: https://orcid.org/0000-0001-8466-4880

Universidade Federal de Campina Grande, Brasil E-mail: jankalmeida@gmail.com

Francisco Paulo de Andrade Alves ORCID: https://orcid.org/0000-0003-1845-2550

Universidade Federal de Campina Grande, Brasil E-mail: franciscoj007bond@gmail.com

Rosângela Vidal de Negreiros

ORCID: https://orcid.org/0000-0001-7242-6447

Universidade Federal de Campina Grande, Brasil

E-mail: rosangelavidaldenegreiros1@gmail.com

Andréia Oliveira Barros Sousa

ORCID: https://orcid.org/0000-0001-9877-1070

Universidade Federal de Campina Grande, Brasil E-mail: andreiabarros2@hotmail.com

Daniela Moura dos Reis Braga

ORCID: https://orcid.org/0000-0002-9737-285X

Universidade Federal de Campina Grande, Brasil E-mail: danimourareis@gmail.com

Ana Beatriz Gouveia de Araújo ORCID: https://orcid.org/0000-0003-3537-3707 Universidade Federal do Rio Grande do Norte, Brasil E-mail: bia_araujo38@hotmail.com

Tatiane Lima de Araújo Silva

ORCID: https://orcid.org/0000-0002-8541-9067

Prefeitura Municipal de Campina Grande, Brasil E-mail: jankalmeida@gmail.com

Elvis Costa Crispiniano

ORCID: https://orcid.org/0000-0002-3404-5157

Hospital Universitário Alcides Carneiro, Brasil E-mail: jankalmeida@gmail.com

Marcos Garcia Costa Morais

ORCID: https://orcid.org/0000-0002-0102-9951

Universidade Estadual da Paraíba, Brasil

E-mail: nutrimarcosgarcia@gmail.com

André Souza Oliveira Albuquerque Viana ORCID: https://orcid.org/0000-0002-3353-5170 Centro Universitário Maurício de Nassau, Brasil E-mail: colecionismo8090@gmail.com

\section{Resumo}

Objetivo: Identificar na literatura a realidade vivenciada pelos enfermeiros no contexto da classificação de risco nos serviços de urgência e emergência. Metodologia: Estudo teórico do tipo revisão integrativa da literatura (RIL), com a finalidade de reunir e sintetizar resultados de estudos já realizados. A pesquisa foi realizada por pares, entre os meses 
de Julho e Setembro de 2021, na Biblioteca Virtual em Saúde (BVS), com o uso dos descritores: Serviços de Urgência e Emergência e Classificação de Risco. A população da pesquisa incluiu 210 documentos; posteriormente foi realizada leitura crítica e reflexiva dos títulos e dos resumos, e correlação com a questão norteadora; assim, estabeleceu-se a amostra de 14 artigos. Resultados: Os enfermeiros são profissionais que adquirem em sua formação as as competências e habilidades necessárias para a triagem e classificação de risco, por possuírem uma linguagem clínica orientada para os sinais e os sintomas apresentados pelo usuário, e não para os diagnósticos, necessariamente, conseguindo estabelecer uma relação de empatia, que é fundamental na minimização de sentimentos como ansiedade, agressividade ou impaciência, e explicando calmamente ao utente o objetivo do processo de triagem. Conclusão: $\mathrm{O}$ enfermeiro é tido como figura primordial nesse processo, de modo que é reiterada a necessidade de capacitações e treinamentos, a fim de uniformizar ao máximo possível a classificação de risco atribuída por eles. Evidenciou-se ainda que para garantia e continuidade do cuidado, faz-se imperativo a aplicação da Sistematização da Assistência em Enfermagem.

Palavras-chave: Enfermeiro; Classificação de risco; Urgência e emergência.

\begin{abstract}
Objective: To identify in the literature the reality experienced by nurses in the context of risk classification in urgent and emergency services. Methodology: Theoretical study of the integrative literature review (RIL) type, with the purpose of gathering and synthesizing the results of studies already carried out. The research was carried out by pairs, between the months of July and September 2021, in the Virtual Health Library (VHL), using the descriptors: Urgency and Emergency Services and Risk Classification. The research population included 210 documents; later, a critical and reflective reading of the titles and abstracts was carried out, and correlation with the guiding question; thus, a sample of 14 articles was established. Results: Nurses are professionals who acquire, in their training, the skills and abilities necessary for risk screening and classification, as they have a clinical language oriented to the signs and symptoms presented by the user, and not to the diagnoses, necessarily, achieving establishing an empathetic relationship, which is essential in minimizing feelings such as anxiety, aggression or impatience, and calmly explaining to the user the purpose of the screening process. Conclusion: The nurse is seen as a key figure in this process, so that the need for training and training is reiterated, in order to standardize as much as possible the risk classification assigned by them. It was also evidenced that to guarantee and continuity of care, it is imperative to apply the Systematization of Nursing Care.
\end{abstract}

Keywords: Nurse; Risk rating; Urgency and emergency.

\begin{abstract}
Resumen
Objetivo: Identificar en la literatura la realidad vivida por los enfermeros en el contexto de la clasificación de riesgo en los servicios de urgencias y emergencias. Metodología: Estudio teórico del tipo revisión integradora de la literatura (EIR), con el propósito de recopilar y sintetizar los resultados de estudios ya realizados. La investigación se realizó por parejas, entre los meses de julio y septiembre de 2021, en la Biblioteca Virtual en Salud (BVS), utilizando los descriptores: Servicios de Urgencias y Emergencias y Clasificación de Riesgos. La población de investigación incluyó 210 documentos; posteriormente, se realizó la lectura crítica y reflexiva de los títulos y resúmenes, y la correlación con la pregunta orientadora; así, se estableció una muestra de 14 artículos. Resultados: Los enfermeros son profesionales que adquieren, en su formación, las destrezas y habilidades necesarias para el cribado y clasificación de riesgos, ya que cuentan con un lenguaje clínico orientado a los signos y síntomas que presenta el usuario, y no a los diagnósticos, logrando, logrando establecer un empático, que es fundamental en relaciones mínimas como la ansiedad, la agresión o la impaciencia, y que explica con calma al usuario el propósito del proceso de selección. Conclusión: El enfermero es visto como una figura clave en este proceso, por lo que se reitera la necesidad de capacitación y entrenamiento, con el fin de estandarizar al máximo la clasificación de riesgo asignada por ellos. También se evidenció que para garantizar la continuidad de la atención es imperativo aplicar la Sistematización de la Atención de Enfermería.
\end{abstract}

Palabras clave: Enfermera; Calificación de riesgo; Urgencia y emergencia.

\title{
1. Introdução
}

Atualmente, o sistema de atenção à saúde no território brasileiro está estruturado em níveis de baixa complexidade (atenção primária), média complexidade (atenção secundária) e alta complexidade e tecnologias (atenção terciária), que têm como objetivo organizar os serviços de atenção à saúde e assim, garantir a integralidade do cuidado. Neste sentido, a porta de entrada do usuário no sistema é a atenção primária, estruturada como primeiro nível de atenção para garantir atendimento das necessidades de saúde de todos os usuários do sistema (Marques et al., 2018).

Entretanto, devido à falta de agilidade e baixa resolutividade dos serviços de atenção básica, à população com menor renda demonstra baixa adesão aos serviços oferecidos nesse nível de atenção, preferindo usar atendimento para suas necessidades de saúde nos Serviços de Urgência e Emergência (SUE), como alternativa para conseguir atendimento imediato e em menor prazo 
(Marques et al., 2018).

O Sistema de Saúde no Brasil encontra-se estruturado em atenção à saúde, de básica, média e alta complexidade. Todos os componentes devem participar da atenção às urgências. No âmbito de atenção às urgências, o Acolhimento com Avaliação e Classificação de Risco (AACR), é uma tecnologia utilizada pelo ministério da saúde no sentido de reorientação da política assistencial nos serviços de emergências, articulando os valores de humanização e qualificação da assistência (Morelato et al., 2021).

Os sistemas de triagem têm o objetivo de organizar a demanda de pacientes no que refere ao atendimento prioritário, nos serviços de urgências, diferenciando os que pacientes que necessitam de atendimento imediato dos que podem esperar em segurança pelo atendimento, antes que haja a avaliação diagnóstica e terapêutica completa (Dias et al., 2018).

O serviço de acolhimento acima descrito, como proposto pela Política Nacional de Humanização (PNH), surgiu como estratégia resolutiva e necessária para aprimorar o atendimento e o acesso aos usuários dos serviços de urgência e emergência. Assim, o acolhimento por si só, tem como principal objetivo incluir e estabelecer relação com os que buscam atendimento nos serviços de baixa, média e alta densidades tecnológicas. Por isso, a avaliação com classificação de risco tem como meta conhecer as prioridades, e assim, avaliar o risco de cada usuário, seguindo protocolo previamente estabelecido e deve ser realizada, necessariamente, pelo enfermeiro (Marques et al., 2018).

Juntos, acolhimento e ACR constituem ferramenta capaz de melhorar o atendimento e reduzir o tempo de espera dos usuários, com a proposta de reorganizar os serviços de urgência e emergência, inovando suas práticas gerenciais e assistenciais para superar os desafios do atendimento aos usuários. Dentre tais desafios, destacam-se de maneira predominante, a superlotação, a desumanização da assistência e a nova ordem dos atendimentos de acordo com o quadro apresentado pelo usuário (Dias et al., 2018).

Destarte, os enfermeiros têm sido os profissionais recomendados para atuar na classificação de risco e tomar decisão acerca do nível de prioridade clínica mediante avaliação e consulta de enfermagem. Assim, através da utilização de protocolos sistematizados, são obtidas informações clínicas por meio de dados objetivos e subjetivos do estado de saúde apresentado pelo paciente, para assim assegurar a priorização e organização do atendimento de maneira resolutiva (Duro et al., 2017).

Neste sentido, considerando que a classificação de risco busca a otimização do fluxo de atendimento a partir da qualificação do atendimento ofertado na porta de entrada dos serviços de urgência, os enfermeiros possuem a responsabilidade de regular o ingresso do usuário no serviço de urgência e emergência. Dado este motivo, os enfermeiros que tomam decisões na triagem devem ter uma base de conhecimento diversificada e, neste sentido, competências e habilidades para avaliação e priorização clínica do paciente (Spagnuolo et al., 2017).

No contexto do sistema único de saúde, mediante às diversas situações em que se encontram as unidades de urgência, a identificação dos problemas enfrentados no processo de acolhimento e classificação de risco poderá ser utilizada como embasamento para qualificar o atendimento prestado, garantindo a segurança do paciente e a organização do serviço. Concomitantemente, o conhecimento sobre o modo pelo qual os enfermeiros desenvolvem a avaliação clínica do paciente implica em tornar a classificação de risco não somente uma seleção de pacientes, mas uma prática clínica destes profissionais no exercício cotidiano da assistência prestada em serviços de urgências (Duro et al., 2017).

No âmbito nacional, as pesquisas já realizadas têm destacado a avaliação da eficácia e qualidade da classificação de risco e seu impacto na dinâmica da organização dos serviços de urgência, assim como a confiabilidade e validade da aplicação do protocolo de Manchester. No entanto, ainda há escassa literatura enfatizando o trabalho executado pelos enfermeiros na classificação de risco diante das dificuldades e vulnerabilidades deste cargo de trabalho. Evidencia-se que ainda há definições a serem estabelecidas para a atuação dos enfermeiros, a fim de sanar as necessidades observadas na área de acolhimento e 
classificação de risco nos serviços de urgência e emergência (Morelato et al., 2021).

Tendo em vista que, nos serviços em questão, o desempenho desse profissional envolve especificidades e articulações indispensáveis à gerência do cuidado a pacientes com necessidades complexas, o que requer aprimoramento científico, manejo tecnológico e humanização extensiva, tem-se como pergunta de pesquisa: Qual a realidade prática vivenciada pelos enfermeiros no âmbito da classificação de risco nos serviços de saúde de urgência e emergência?

Assim ,o objetivo geral desta pesquisa é identificar na literatura a realidade vivenciada pelos enfermeiros no contexto da classificação de risco nos serviços de urgência e emergência. Os objetivos específicos são: evidenciar os desafios observados na atuação da classificação de risco nos serviços de urgência e emergência e apresentar a atuação profissional dos enfermeiros no contexto da classificação de risco em serviços de urgência e emergência.

\section{Metodologia}

Estudo teórico do tipo revisão integrativa da literatura (RIL), com a finalidade de reunir e sintetizar resultados de estudos já realizados, e contribuir com o aprofundamento do conhecimento relativo ao tema investigado.

A RIL permeou as etapas preconizadas pelo Joanna Briggs Institute (JBI, 2011) para construção do protocolo de pesquisa: formulação da questão norteadora para a elaboração da pesquisa, utilizando a estratégia PICO; especificação dos métodos de seleção dos estudos; procedimento de extração dos dados; análise e avaliação dos documentos incluídos na pesquisa; extração dos dados e apresentação da revisão/síntese do conhecimento produzido e publicado.

Os problemas clínicos que surgem na prática assistencial, de ensino ou pesquisa, são decompostos e, a seguir, organizados utilizando-se a estratégia PICO.

PICO representa um acrônimo para Paciente, Intervenção, Comparação e "Outcomes" (desfecho). Esses quatro componentes são os elementos fundamentais da questão de pesquisa e da construção da pergunta para a busca bibliográfica de evidências (Akobeng, 2009. p 837) A estratégia PICO pode ser utilizada para construir questões de pesquisa de naturezas diversas, oriundas da clínica, do gerenciamento de recursos humanos e materiais, da busca de instrumentos para avaliação de sintomas, entre outras (Bernardo, Nobre \& Jatene, 2004). Pergunta de pesquisa adequada (bem construída) possibilita a definição correta de que informações (evidências) são necessárias para a resolução da questão clínica de pesquisa, maximiza a recuperação de evidências nas bases de dados, foca o escopo da pesquisa e evita a realização de buscas desnecessárias.

Abaixo está descrito o Quadro 1, com os acrômios e suas respectivas definições, através dos objetivos propostos por esta pesquisa para a melhor definição de sua pergunta de pesquisa. 
Quadro 1. Acrômios e suas respectivas definições.

\begin{tabular}{|c|c|c|}
\hline Acrônimo & Definição & Descrição \\
\hline P & Paciente ou problema & $\begin{array}{r}\text { Desafios vivenciados pelos enfermeiros durante a realização da classificação de risco } \\
\text { nos serviços de Urgência e Emergência. }\end{array}$ \\
\hline I & Intervenção & $\begin{array}{r}\text { Realização da pesquisa na literatura e nas bases de dados, midiatização do } \\
\text { conhecimento científico, melhoria das condições de trabalho. }\end{array}$ \\
\hline C & Controle ou Comparação & $\begin{array}{r}\text { Melhoria das condições de trabalho, maior suporte por parte da gestão, melhores } \\
\text { condições estruturais e de ordem laboral. }\end{array}$ \\
\hline O & Desfecho (“Outcomes") & $\begin{array}{r}\text { Observação da realidade prática dos enfermeiros no intuito de promover melhorias } \\
\text { nas condições de trabalho e melhor enfrentamento das dificuldades laborais. }\end{array}$ \\
\hline
\end{tabular}

Fonte: Autor.

Assim, através da descrição dos fatores acima, foi definida como pergunta de pesquisa: quais os desafios vivenciados pelos enfermeiros durante a classificação de risco nos serviços de Urgência e Emergência?

A partir disto, a pesquisa foi realizada por pares, entre os meses de Julho e Setembro de 2021, na Biblioteca Virtual em Saúde (BVS), com o uso dos descritores de busca controlada Serviços de Urgência e Emergência e Classificação de Risco, associando-se aos operadores booleanos "and", e selecionando amostra a partir dos filtros texto completo e disponível, documentos tipo artigos científicos; ano de publicação 2013 a 2021; e sem distinção de idioma.

A população da pesquisa incluiu 210 documentos; os critérios de inclusão utilizados foram texto completo e disponível, artigos cujo assunto principal respondia à pergunta de pesquisa proposta e aos objetivos do trabalho. Como critérios de exclusão, textos incompletos, artigos do tipo revisão, artigos que fugiram do tema principal proposto por esta pesquisa. Posteriormente foi realizada leitura crítica e reflexiva dos títulos e dos resumos, e correlação com a questão norteadora. Assim, estabeleceu-se a amostra de 14 artigos.

Para extração dos dados dos documentos selecionados, fez-se necessária a utilização de um instrumento previamente elaborado, que fosse capaz de assegurar que a totalidade dos dados relevantes fosse extraída, minimizando o risco de fragilidades/erros na transcrição, garantindo precisão na checagem das informações e servindo como registro. Para isto utilizouse o instrumento de coleta de dados validado por Ursi (2005) para elaboração de protocolo próprio para esta pesquisa. Em fase sequente, os dados secundários foram organizados a partir das respostas à questão norteadora do estudo, em categorias temáticas e posteriormente discutidos a partir da análise de conteúdo Bardin (2011), com respaldo da literatura científica pertinente.

Em seguida, na etapa analítica, três fases foram sequenciadas. A primeira foi a Pré-análise, com a exploração do material e tratamento dos resultados. Nesta, realizou-se a "leitura flutuante", portanto, a inicial leitura dos documentos selecionados, organizando os indicadores de interpretação como conteúdos norteadores encontrados na leitura completa dos artigos. Na segunda fase, de exploração do material, observaram-se os temas que se repetiam nos artigos para elaboração das categorias iniciais; isto é, as unidades de codificação, classificação e categorização. Na terceira fase concretizou-se o tratamento dos resultados, através da inferência e interpretação destes, discutidos a seguir, de acordo com cada categoria definidora.

\section{Resultados e Discussão}

Embora a necessidade de melhorias na qualidade do atendimento em serviços de emergência seja reconhecida, os meios para tal feito são complexos por dependerem de um conjunto coordenado de ações que incluem maior resolutividade dos casos 
pela rede básica de saúde; integração entre as redes pública e privada; contratos de regulação adequados, baseados num modelo assistencial responsivo às necessidades de saúde dos usuários e profissionais; e também, a existência de um conjunto tão completo quanto possível, de protocolos e dispositivos de atendimento, tal como o Acolhimento com Classificação de Risco, proposto pelo Ministério da Saúde, no nosso sistema de atenção à saúde (Caveião et al., 2014).

Nesta perspectiva, os serviços de emergência são considerados importantes componentes do Sistema de Saúde, pois atendem usuários que são afetados por comorbidades de natureza aguda, com ou sem risco iminente de morte. Neste sentido, para melhorar a qualidade do atendimento em atendimentos de emergência, o SUS propõe diretrizes que se referem à definição de protocolos clínicos, à criação de mecanismos de referência e contrarreferência e ao acolhimento da demanda por meio de critérios de classificação e avaliação de risco (Feitosa et al., 2017).

Atualmente se observa que a implantação do dispositivo Acolhimento com Classificação e Avaliação de Risco (ACCR) em serviços de emergência tem apresentado resultados positivos no que diz respeito ao controle do fluxo de atendimentos e à priorização dos agravos para o melhor direcionamento do usuário (Oliveira et al., 2016).

Na definição para o ACCR, trazido por Júnior \& Matsuda (2013), o Ministério da Saúde estabelece que esse dispositivo consiste em uma diretriz operacional que unifica as ações de acolhimento com as de classificação de risco do usuário. Isso quer dizer que, no ACCR, o usuário que se dirige ao serviço de emergência é acolhido, ouvido, encaminhado à consulta de enfermagem, classificado conforme o grau de risco de seu agravo e atendido pelo médico de acordo com a urgência do caso. As ações de acolhimento ao usuário podem ser realizadas por qualquer profissional da equipe de saúde, desde que treinado para isto; entretanto, cabe ao enfermeiro o papel de classificar e avaliar o risco do paciente/cliente/usuário, de acordo com o grau de urgência de seu agravo, com base em um sistema de cores predefinido: vermelho = emergência; amarelo = urgência; verde = menor urgência; azul = não urgência (Roncalli et al., 2017).

A situação dos serviços de urgência e emergência tem recebido mais atenção dos governantes e da sociedade. A procura por esses serviços tem sido cada vez mais frequente devido ao aumento do número de acidentes e da violência urbana. Além dessa demanda, muitos dos atendimentos são decorrentes de doenças de baixa complexidade, encaminhados para esse serviço, por insuficiente estruturação da rede básica, como descrito (Dias et al., 2018).

No contexto dos serviços de urgência e emergência, onde os mesmos se deparam com grandes filas de espera e sem critérios, exceto o da hora de chegada, a distinção de riscos ou graus de sofrimentos se faz necessário para que todos sejam acolhidos. A adoção de um sistema de classificação de risco no acolhimento assegura o atendimento de acordo com a condição de saúde, apresentada na avaliação inicial realizada pelo profissional enfermeiro na triagem. Além disso, o paciente que não corre risco imediato, assim como a seus familiares, são informados sobre o tempo provável de espera (Morelato et al., 2021).

Nessa atividade, a triagem apresenta-se como um trabalho essencial para o correto redirecionamento dos pacientes, ao mesmo tempo em que tem como finalidade descongestionar o serviço de urgência e emergência. No entanto, deve ser realizada por profissionais qualificados, aumentando a resolutividade dos problemas e a qualidade do atendimento (Spagnuolo et al., 2017). É sabido que essa atividade, recém-implantada, é realizada por enfermeiros capacitados que, juntamente com o médico, constituem a equipe dos serviços de emergência. Vários estudos científicos têm sido realizados com o objetivo de compreender se existem benefícios na realização de triagem por enfermeiros ou médicos. No entanto, a literatura tem demonstrado que o enfermeiro é o profissional de saúde mais apto para o desempenho dessa atividade, tornando esta uma atividade privativa da profissão (Spagnuolo et al., 2017).

Os enfermeiros são profissionais que adquirem em sua formação as as competências e habilidades necessárias para a triagem e classificação de risco, por possuírem uma linguagem clínica orientada para os sinais e os sintomas apresentados pelo usuário, e não para os diagnósticos, necessariamente, conseguindo estabelecer uma relação de empatia, que é fundamental na 
minimização de sentimentos como ansiedade, agressividade ou impaciência, e explicando calmamente ao utente o objetivo do processo de triagem. Para além disso, os enfermeiros têm uma visão da globalidade do serviço e dos recursos neles existentes (Oliveira et al., 2016).

Para o desenvolvimento da Classificação de Risco (CR), os enfermeiros utilizam a escuta qualificada das queixas e das condições de saúde do paciente para a identificação do problema que motivou a busca pelo serviço de urgência. A partir da escuta ativa, o enfermeiro identifica o risco e a vulnerabilidade e acolhe a avaliação do próprio paciente, para tomar uma decisão na CR.Neste sentido, a escuta de informações e a forma com que os pacientes referem seus problemas aos enfermeiros da triagem são sinalizadas como ponto de partida para a tomada de decisão e a classificação clínica dos pacientes (Duro et al., 2017).

Assim, na avaliação da situação clínica do paciente, a escuta das queixas aliada ao compromisso de dar respostas às necessidades de saúde trazidas pelos usuários, consiste em habilidade importante dos enfermeiros no processo de CR. Aliada à escuta, outra potencialidade da CR é o uso do saber clínico na identificação do risco de acordo com a condição de saúde do paciente. Os estudos trazidos demonstram a especificidade de informações clínicas obtidas quando o processo de triagem inicia e como isso se relaciona com a classificação de risco do paciente. A partir disso, infere-se que os cuidados prestados no serviço de emergência estão significativamente relacionados com a precisão na identificação de problemas e sua interação no processo de tomada de decisão clínica na CR (Acosta et al., 2013).

O enfermeiro responsável pela CR busca informações relevantes sobre o paciente, sendo esse um processo contínuo, o qual necessita de uma combinação de informações, conhecimento clínico e julgamento deste profissional. Dessa forma, a atividade dos enfermeiros na $\mathrm{CR}$ vem sendo descrita como complexa, não consistindo apenas no resultado de uma avaliação para a determinação do risco baseada em protocolos profissionais, mas na maneira como os enfermeiros desenvolvem estratégias para a tomada de decisão na priorização do atendimento (Acosta et al., 2013; Mendonça et al., 2018).

A classificação de risco é um processo dinâmico de identificação de pacientes que necessitam de tratamento imediato. Isto é desenvolvido de acordo com o potencial de risco, agravo à saúde, ou grau de sofrimento, devendo o atendimento ser priorizado conforme a gravidade clínica do paciente e não com a ordem de chegada ao serviço. Corroborando com este fato já citado, o processo de adaptação da queixa do paciente ao fluxograma do protocolo é um dos processos mais difíceis, pois exige deste profissional escuta qualificada, avaliação e registro correto e detalhado da queixa principal, capacidade de observação, raciocínio clínico, tomada de decisão e conhecimento das redes de apoio do sistema assistencial disponíveis (Morelato et al., 2021).

Ainda, o fluxograma no qual a queixa do paciente é direcionada e sequencialmente classificada, muitas vezes não se correlaciona com o relato do paciente. Isto requer uma habilidade de entendimento e interpretação maior por parte do enfermeiro que realiza a classificação, sendo assim é fundamental a análise do enfermeiro, tendo uma visão clínica e mais profunda da queixa do usuário. Desta maneira, é fundamental que o enfermeiro classificador reconheça a educação permanente como aliada ao longo da vida profissional, com vistas a adquirir e manter um lastro de conhecimento que possa embasar seu raciocínio clínico no processo de classificação (Hermida et al., 2017).

A educação permanente quando estimulada visa a valorização dos enfermeiros que atuam com ACCR, pois proporciona maior integração entre os trabalhadores e os usuários do sistema de saúde. Envolvem a articulação entre educação e trabalho no SUS, visando à produção de mudanças nas práticas de formação e de saúde, pois permite o desenvolvimento da capacidade pedagógica de problematizar e identificar pontos sensíveis e estratégicos para a produção da integralidade e humanização (Roncalli et al., 2017).

Nessa linha de raciocínio, é sabido que existem inúmeros desafios no contexto da assistência em saúde nos serviços de urgência e emergência, em que, por exemplo, os profissionais de saúde se deparam com carga horária exorbitante, mau 
dimensionamento e má distribuição/déficit de profissionais, inúmeras atribuições que fogem das competências de sua classe, além da falta de materiais e insumos, superlotação dos serviços e alta complexidade das demandas, sendo estes os principais entraves trazidos pelos estudos que embasam esta pesquisa, demonstrando a necessidade de reorganização e de uma gestão técnica e qualificada, que traz consigo soluções viáveis e resolutivas para atender às necessidades específicas deste serviço (Hermida et al., 2017).

\section{Conclusão}

Apesar dos avanços e conquistas, a implantação do AACR nos serviços de urgência e emergência ainda está em construção, necessitando avaliações contínuas para identificar e corrigir falhas para oferecer melhorias na qualidade dos serviços disponibilizados.

O enfermeiro é tido como figura primordial nesse processo, de modo que é reiterada a necessidade de capacitações e treinamentos, a fim de uniformizar ao máximo possível a classificação de risco atribuída por eles. Evidenciou-se ainda que para garantia e continuidade do cuidado, faz-se imperativo a aplicação da Sistematização da Assistência em Enfermagem.

Assim, destaca-se também à necessidade de buscar e criar os pontos de atenção suficientes para dar soluções resolutivas às demandas e necessidades dos usuários, tentando ampliar a resolutividade do sistema assistencial de saúde, práticas de acolhimento em todos os pontos de atenção, grande ampliação das instalações para maior capacidade, organização de ações e do sistema de regulação, controle e avaliação, ações que incluam a participação dos profissionais com o intuito de provocar mudanças de práticas na relação com os usuários, e adoção de mecanismos e fluxos de encaminhamentos de pacientes entre os serviços.

Nesse sentido, o enfermeiro é peça fundamental no funcionamento eficiente deste serviço, pois a classificação de risco é responsabilidade específica deste profissional. É ele quem realiza o julgamento clínico e crítico das queixas e, a partir das informações cedidas pelos usuários, determinará o risco e a ordem de atendimento para cada caso. Contudo, para este fim, o enfermeiro deve conhecer todas as etapas do processo e atuar de maneira holística. Além disso, deve observar a abrangência do ACCR, desconstruindo a ideia de que se trata apenas de um local ou parte do atendimento. Apesar das dificuldades e carências que fragilizam o trabalho do enfermeiro nesse processo, o conhecimento acerca da ausculta qualificada, julgamento clínico e crítico de exames sucintos, assim como, análise psicológica, interpessoal de cada paciente favorece o julgamento apropriado de cada caso influenciando diretamente na sua eficiência e eficácia do ACR.

Ainda considerando que as mudanças políticas e organizacionais, geralmente, são propostas para melhorar os serviços ofertados aos usuários, é fundamental conhecer a opinião destes sobre a qualidade do atendimento recebido. $\mathrm{O}$ respeito às expectativas e necessidades dos usuários e sua satisfação com o atendimento recebido nos serviços de saúde direciona o planejamento em saúde e é indispensável para repensar as práticas assistenciais e gerenciais dos serviços. Além disso, estudos de avaliação da satisfação constituem ferramenta para a participação da comunidade que usa o serviço.

Este estudo tem o intuito de contribuir para a melhoria dos serviços de emergência de uma maneira geral, através da avaliação e levantamento das evidências científicas advindas das práticas em saúde neste mesmo contexto. Assim, como sugestões para o desenvolvimento de estudos futuros, é de suma importância que se busque a avaliação rotineira dos serviços de saúde, que contribui para o fortalecimento do SUS, como também, do usuário como objeto central do trabalho dos profissionais. A avaliação de políticas, programas e serviços de saúde possibilita a reorientação dos planejamentos, das ações e das estratégias usadas para alcançar o êxito do atendimento oferecido. 


\section{Referências}

Acosta, A. M., Duro, C. L. M., \& Lima, M. A. D. D. S. (2012). Atividades do enfermeiro nos sistemas de triagem/classificação de risco nos serviços de urgência: revisão integrativa. Revista gaúcha de enfermagem, 33, 181-190.

Akobeng, A. K. (2005). Princípios da medicina baseada em evidências. Arquivos de doenças na infância, v. 90, n. 8, pág. 837-840.

Bardin, L. (2011). Análise de conteúdo. 4a ed. Lisboa: Almedina.

Bernardo, W. M., Nobre, M. R. C., \& Jatene, F. B. (2004). A prática clínica baseada em evidências: parte II-buscando as evidências em fontes de informação. Revista Brasileira de Reumatologia, 44, 403-409.

Caveião, C., Hey, A. P., Montezeli, J. H., Barros, A. P. D. M. M., Sordi, J. A., \& Santos, S. C. (2014). Desafios ao enfermeiro na implantação da classificação de risco em unidade mista. Revista de Enfermagem da UFSM, 4(1), 189-196.

Chianca, T. C. M., Costa, R. D. M., Vidigal, M. V., Silva, L. C. R. D., Diniz, G. A., Araújo, J. H. V., \& Souza, C. C. D. (2016). Tempos de espera para atendimento usando Sistema de Triagem de Manchester em um hospital de urgência. Revista Mineira de Enfermagem, v 20.

Dias, S. R. S., Santos, L. L., \& Silva, I. A. (2018). Classificação de risco no serviço de urgência e emergência: revisão integrativa da literatura. Rev. enferm. UFPI, 57-62.

Duro, C. L. M., Lima, M. A. D. S., \& Weber, L. A. F. (2017). Nurses' opinion risk classification in emergency services. Rev Min Enferm [Internet], 21 , e-1062.

Feitosa, M. M., Silva, I. C. G., Costa, R. T. D. S., Andrade, I. C. F. D., Neto, V. L. D. S., \& Silva, R. A. R. D. (2017). Acolhimento com classificação de risco na unidade de pronto atendimento: um relato de experiência. Rev. enferm. UFSM, 1-8.

Hermida, P. M. V., Jung, W., do Nascimento, E. R. P., Silveira, N. R., Alves, D. L. F., \& Benfatto, T. B. (2017). Classificação de risco em unidade de pronto atendimento: discursos dos enfermeiros [Risk classification in an emergency care unit: the nurses' discourse][Clasificación de riesgo en unidad de urgencias: discursos de los enfermeros]. Revista Enfermagem UERJ, 25, 19649.

Bellucci, J., \& Matsuda, L. M. (2012). Implantação do acolhimento com classificação de risco em serviço hospitalar de emergência: atuação do enfermeiro. Ciência, Cuidado e Saúde, 11(2), 396-401.

Marques, L. A., César, F. C. R., de Resende Izidoro, L. C., Cabral, K. B., Santos, L. F., Brasil, V. V., \& Oliveira, L. M. D. A. C. (2018). Satisfação de usuários com o acolhimento e classificação de risco em unidades públicas de saúde. Revista Eletrônica de Enfermagem, v 20.

Mendonça, A. R., de Carvalho Queluci, G., Rodrigues de Souza, V., Couto Dias, S. F. \& da Silveira Jasmim, J. (2018). Habilidades de enfermagem em serviços de emergência. Revista de Enfermagem UFPE. 12 (10).

Morelato, C. S., Dorneles, L. L., Martins, V. D. P., Goés, F. D. S. N. D., Viana, A.L, Brunello, M. E. F. \& Camargo, R. A. A. D. (2021). Recebendo demanda espontânea na Atenção Básica: necessidades de aprendizagem do enfermeiro. Revista Brasileira de Enfermagem, v 74.

de Oliveira, J. L. C., de Souza, V. S., Inoue, K. C., Costa, M. A. R., Camillo, N. R. S., \& Matsuda, L. M. (2016). < b> Atuação do enfermeiro no Acolhimento com Classificação de Risco: um estudo de metassíntese/Nurse's practice in Welcoming with Risk Classification: a meta-synthesis study. Ciência, Cuidado e saúde, 15(2), 374-382.

Roncalli, A. A., Oliveira, D. N., Silva, I. C. M., Brito, R. F., \& Viegas, S. M. F. (2017). Manchester protocol and user population in the risk assessment: the nurse's view. Rev baiana enferm, 31(2), e16949.

de Souza, C. C., Chianca, T. C. M., Júnior, W. C., Rausch, M. D. C. P., \& Nascimento, G. F. L. (2018). Análise da confiabilidade do Sistema de Triagem de Manchester: concordância interna e entre observadores. Revista Latino-Americana de Enfermagem, 26, e3005-e3005.

Spagnuolo, R. S., Silva, M. N. L., Meneguin, S., Bassetto, J. G. B., \& Fernandes, V. C. (2017). Percepção dos usuários sobre a triagem com classificação de risco em um serviço de urgência de cabo verde. Revista Brasileira em Promoção da Saúde, 30(2), 249-254.

Ursi, E. S., \& Gavão, C. M. (2006). Prevenção de lesões de pele no perioperatório: revisão integrativa da literatura. Revista Latino-Americana de Enfermagem, 14, 124-131. 